\title{
ABBaH teens: Activity Breaks for Brain Health in adolescents: study protocol for a randomized crossover trial
}

\author{
Emerald G. Heiland ${ }^{1,2^{*}}$ (D), Karin Kjellenberg ${ }^{1}$, Olga Tarassova ${ }^{3}$, Maria Fernström', Gisela Nyberg ${ }^{1,4}$, Maria M. Ekblom,5,
} Björg Helgadottir ${ }^{1,6}$ and Örjan Ekblom ${ }^{1}$

\begin{abstract}
Background: Physical activity breaks are widely being implemented in school settings as a solution to increase academic performance and reduce sitting time. However, the underlying physiological mechanisms suggested to improve cognitive function from physical activity and the frequency, intensity, and duration of the breaks remain unknown. This study will investigate the effects of frequent, short physical activity breaks during prolonged sitting on task-related prefrontal cerebral blood flow, cognitive performance, and psychological factors. Additionally, the moderating and mediating effects of arterial stiffness on changes in cerebral blood flow will be tested.

Methods: This is a protocol for a randomized crossover study that will recruit 16 adolescents (13-14 years old). Participants will undergo three different conditions in a randomized order, on three separate days, involving sitting 80 min with a different type of break every $17 \mathrm{~min}$ for $3 \mathrm{~min}$. The breaks will consist of (1) seated social breaks, (2) simple resistance activities, and (3) step-up activities. Before and after the 80-min conditions, prefrontal cerebral blood flow changes will be measured using functional near-infrared spectroscopy (primary outcome), while performing working memory tasks (1-, 2-, and 3-back tests). Arterial stiffness (augmentation index and pulse wave velocity) and psychological factors will also be assessed pre and post the 80-min interventions.

Discussion: Publication of this protocol will help to increase rigor in science. The results will inform regarding the underlying mechanisms driving the association between physical activity breaks and cognitive performance. This information can be used for designing effective and feasible interventions to be implemented in schools.
\end{abstract}

Trial registration: www.ClinicalTrials.gov, NCT04552626. Retrospectively registered on September 21, 2020.

Keywords: Physical activity breaks, Sedentary, Cerebral blood flow, Cognitive function, fNIRS

\section{Background}

Implementation of classroom-based physical activity breaks is being considered as a potential solution to combating sedentary behavior among adolescents and improving cognitive function and scholastic performance

\footnotetext{
* Correspondence: emerald.heiland@gih.se

'Department of Physical Activity and Health, The Swedish School of Sport and Health Sciences $(\mathrm{GIH})$, Lidingövägen 1, 11433 Stockholm, Sweden

2Department of Surgical Sciences, Medical Epidemiology, Uppsala University, Dag Hammarskjölds väg 14B, 75185 Uppsala, Sweden

Full list of author information is available at the end of the article
}

[1]. Focus on adolescents is pivotal as they are among the least studied groups in relation to physical activity and cognitive performance [2, 3]. Additionally, in Sweden, adolescents spend about $10.3 \mathrm{~h}$ per day sedentary, and only $43 \%$ of boys and $23 \%$ of girls meet the physical activity recommendations [4]. However, previous research on the effects of physical activity breaks on cognitive performance in adolescents has been inconclusive, mainly due to heterogeneity in study designs and methodology [1, 5-9].

C C The Author(s). 2022 Open Access This article is licensed under a Creative Commons Attribution 4.0 International License, which permits use, sharing, adaptation, distribution and reproduction in any medium or format, as long as you give appropriate credit to the original author(s) and the source, provide a link to the Creative Commons licence, and indicate if changes were made. The images or other third party material in this article are included in the article's Creative Commons licence, unless indicated otherwise in a credit line to the material. If material is not included in the article's Creative Commons licence and your intended use is not permitted by statutory regulation or exceeds the permitted use, you will need to obtain permission directly from the copyright holder. To view a copy of this licence, visit http://creativecommons.org/licenses/by/4.0/ The Creative Commons Public Domain Dedication waiver (http://creativecommons.org/publicdomain/zero/1.0/) applies to the data made available in this article, unless otherwise stated in a credit line to the data. 
Particular focus has been given to executive functions [10], as they are potential prerequisites for successful learning [11], emotional regulation [12], and child development [13]. Furthermore, among the cognitive functions, enhancement from physical activity has been most evident in the executive functions [14]. Working memory is a core component of the executive functions and involves holding information in mind and manipulating it to meet task goals [15], but has not been often studied in relation to physical activity nor in younger populations [2]. There is a clear connection between adolescents' physical activity and cognitive ability [5], but knowledge is lacking on which intensity, frequency, and duration of physical activity is necessary to influence physiological mechanisms important for brain health and function.

One important mechanism that may explain the effects of physical activity on cognitive performance is changes in blood circulation in the cerebral cortex, especially in the frontal lobe (prefrontal), which is involved in solving cognitively demanding tasks $[16,17]$, but the majority of research has been in adults. Generally, results have revealed that a single bout of exercise can elicit immediate cognitive task-related increases on cerebral blood flow post exercise cessation [18-22], but also decreases or no effects have been observed [23, 24]. Reasons for differing results are partly related to the moderating effects of timing, duration, and intensity of the physical activity, and the timing and type of cognitive test performed.

Frequent, short physical activity breaks may be a more feasible alternative than a single bout, to counter the negative effects of prolonged sitting during a school day [25]. Studies have demonstrated positive effects of frequent, short physical activity breaks on peripheral vasculature in adults and older adults [26-30], but effects on cerebral vasculature remain elusive, especially in adolescents. In adults, studies performed using frequent, short physical activity breaks have been inconsistent, showing positive, negative, and no effects on cerebral blood flow [31-34] and on cognition [35-38]. Decreases in cerebral blood flow, with favorable effects on cognitive performance, have been seen in adult populations and suggest a potential neural efficiency adaptation [23, 24, 39, 40]. This suggests that after performing physical activity, efficiency in performing the cognitively demanding task increases so that less resources are required [39]. However, it remains unknown if a similar adaptation occurs in adolescents. Furthermore, studies that have measured cerebral blood flow have used indirect measurement modalities, such as transcranial Doppler ultrasound. One study using a more robust modalityfunctional near-infrared spectroscopy (fNIRS) - to study cerebral blood flow, found that male children with autism spectrum disorders had significant beneficial effects of a 20-min physical activity bout, compared to a sedentary counterpart, on prefrontal task-related cerebral blood flow [41]. Although performed in younger children (ages 8-10), another study found that after intermittent or continuous exercise, prefrontaldependent cognitive performance improved compared to the baseline measure and this was significantly explained by alterations in cerebral blood flow assessed using fNIRS [42]. With fNIRS, it is possible to monitor cerebral blood flow by measuring changes in the attenuation of near-infrared light passing through the cortical tissue. Because fNIRS is non-invasive and does not require a certain posture or setting as in functional magnetic resonance imaging (fMRI), it is often used for brain imaging studies in infants and children [13]. It is considered a more feasible and robust option to measure cerebral blood flow changes, particularly when used in conjunction with short-separation channels, to eliminate the contaminating signal from blood flow in other tissues [43]. There are yet no studies investigating the effects of frequent, short physical activity breaks on hemodynamic changes using this technique in adolescents. We believe that the use of short-separation channels will produce partly different results.

Earlier studies have mainly focused on the effects of physical activity breaks only on cognitive performance, neglecting the underlying mechanisms [1]. Two studies have compared how different frequencies and durations of physical activity breaks affect cognitive performance. One study in children found that two 20-min breaks of moderate intensity were better than one or none for cognitive performance [44]. The other study found that 10-20 min long moderate-to-vigorous aerobic bouts were more beneficial for cognitive performance than that of a shorter duration (5 min) [8]. Favorable effects on working memory, as well as on well-being, were also observed in a school setting with a 15 min self-paced physical activity break at intensities near maximal exhaustion (the "Beep test") [45]. Although, according to the inverted- $U$ theory, high-intensity physical activity can produce unfavorable effects on cognitive performance [14]. Moderate-to-vigorous intensity is most favorable on prefrontal cognitive performance, especially when measured immediately after exercise cessation [14]. However, one study of young adults found that breaking up prolonged sitting with a 6-min bout of high-intensity interval exercise had no effect on a prefrontal-dependent cognitive test [46]. Studies incorporating both cerebral blood flow and cognitive performance are needed. A study in adults found that frequent, short walking breaks of moderate-to-vigorous intensity had beneficial effects on working memory after $3 \mathrm{~h}$ of sitting, although, with a coinciding decrease in cerebral blood flow [40]. 
However, it was not significantly different from the uninterrupted sitting condition. Nevertheless, significant improvements were seen in mood and alertness after the walking break condition compared with the uninterrupted sitting [40]. Thus, physical activity breaks may improve psychological health in settings where prolonged sitting occurs, but requires investigation in adolescents and whether favorable effects can also be demonstrated for working memory performance.

In addition, physical inactivity has been found to negatively affect vascular health (arterial stiffness) even among children $[47,48]$, whereas physical activity breaks may counter these effects $[49,50]$. Arterial stiffness can also affect the cerebral vasculature and may therefore moderate or mediate effects of breaking up prolonged sitting on task-related changes in cerebral blood flow.

Furthermore, for activities to be sustainable in a realworld school setting, they need to be inclusive and nondemanding, but also possible to be performed on a regular basis. We therefore sought to investigate the effect of such activities. In turn, the results can reveal not only the potential underpinning mechanisms related to physical activity and working memory performance, but also provide insight into the type, intensity, and duration of physical activity breaks that can be implemented in schools to improve learning and psychological wellbeing throughout the school day.

\section{Aims and research questions}

The main purpose of this study is to investigate how $80 \mathrm{~min}$ of prolonged sitting with and without frequent, short physical activity breaks of different intensities affects fNIRS-measured cerebral blood flow (measured as changes in oxygenated hemoglobin [oxy-Hb]) in the prefrontal cortex, in an ecologically valid mimicked classroom setting, during working memory tests of differential mental workloads in 1314-year-olds.

Our primary research question is:

How does oxy-Hb in the prefrontal cortex during a working memory test change from before to after 80 min of prolonged sitting and after $80 \mathrm{~min}$ of sitting with physical activity breaks; and do these changes differ between these 80-min conditions?

Our secondary questions are:

1. Do physical activity breaks during prolonged sitting affect cognitive performance on the 1-, 2-, and 3-back tests; and self-reported psychological factors (stress, mood, alertness, and sleepiness)? Are these changes significantly different between conditions?

2. Are the changes in cognitive performance correlated with changes in oxy- $\mathrm{Hb}$ ?
3. Does baseline arterial stiffness (measured as augmentation index and pulse wave velocity) moderate the change in cerebral blood flow and cognitive performance?

4. Does the change in arterial stiffness mediate the changes in cerebral blood flow and cognitive performance?

\section{Hypotheses}

We hypothesize that:

a. Task-related increases in oxy-Hb will occur after the conditions involving physical activity breaks and will either stay the same or decrease after the prolonged sitting, and these changes will be significantly greater for the higher intensity break.

b. Cognitive performance, specifically reaction time and accuracy on the n-back tests, will improve after the physically active conditions, but decrease or remain the same after the prolonged sitting condition. The changes will significantly differ between sitting with or without breaks of different intensities, such that more favorable changes will occur when the breaks are of higher intensity.

c. There will be a correlation between changes in oxy$\mathrm{Hb}$ and cognitive performance.

d. Psychological factors, including cortisol-measured stress, and self-reported mood, alertness, and sleepiness, will improve after the physical activity break conditions, which will be significantly different from the prolonged sitting condition, where there will be no change.

e. Arterial stiffness will be reduced after the physically active break conditions, and these changes will be different from the prolonged sitting condition, where no changes will occur or an increase will be observed.

f. Changes in oxy-Hb will be mediated by changes in arterial stiffness.

g. Baseline arterial stiffness will moderate the change in oxy- $\mathrm{Hb}$ and cognitive performance, such that there will be a more pronounced change in those with lower arterial stiffness.

\section{Methods and design Trial design}

A crossover randomized trial with three conditions will be carried out. All procedures will take place at the Swedish School of Sport and Health Sciences (GIH) in Stockholm, Sweden. Initially, each participant will attend a familiarization visit either at the laboratory at GIH with parental accompaniment or the researchers will visit the schools. 
After the familiarization visit, participants will be assigned 3 experimental days for undergoing 3 different randomly ordered experimental conditions, each consisting of an 80-min sitting exposure. There will be a minimum washout period of 7 days between each visit [51, 52], to reduce carryover effects from the previous condition. Two participants will be tested simultaneously (with a 20-min gap between starting times) to increase efficiency and comfort for the participants. Participants will have a 24-h monitoring and standardization of their physical activity, sleep, and dinner prior to each experimental test day. Participants will receive compensation (600 SEK gift card) for their participation.

\section{Participants}

Researchers will initially contact and visit interested schools in Stockholm and the greater Stockholm region, based on schools that have participated in a previous cross-sectional study at $\mathrm{GIH}$, in order to minimize recruitment challenges. After agreement by teachers and the schools' principals, consent forms will be sent home with the students to be signed by both parents prior to data collection. Students will then be invited to come to the laboratory at GIH with parental accompaniment for a familiarization session or researchers will visit the schools to introduce the study.

\section{Eligibility criteria}

Inclusion criteria are children aged 13 to 14 years. Those with ongoing medication that can affect the central or cerebrovascular circulation will be excluded, as well as those who have an ongoing infection, or are unable to apprehend information about the study or how to perform the tests. Students and parents must provide written informed consent prior to study commencement. Participants will be also informed that they may drop out from the study whenever they deem it necessary.

\section{Familiarization visit}

Demographic and other data to describe the individual participant will be collected during the familiarization session including age, gender, height (not if familiarization was performed at the school) and weight without shoes, and a health screening questionnaire for potential contra-indicators. They will also be advised regarding what types of situations during testing that could lead to an immediate discontinuation to ensure safety in the laboratory (i.e., chest pain or similar; difficulties breathing; dizziness, confusion, slowed movements, feelings of sickness, coldness or cold sweaty skin; increase in heart rate not related to increased physical activity intensity; test leader decides to abort; physical or verbal signs of expressed exhaustion; or test equipment breaks). Blood pressure and heart rate will also be measured after $5 \mathrm{~min}$ of rest. In order to optimize fNIRS cap fitting, to increase reproducibility of fNIRS cap placement for each subsequent visit, and for head remodeling in future analyses, head circumference, nasionto-inion, and left and right pre-auricular measurements will be taken, using a tape measure. The cognitive test will also be performed to increase familiarity and reduce practice effects.

A form with choices of food items, provided by Coop Sweden, will also be filled in (i.e., bread [soft or hard]; toppings [butter, cheese, red pepper, or cucumber]; yogurt or sour milk [filmjölk]; muesli or rye cereal; fruit [apple or clementine]), in order to standardize breakfast on experimental days. Additionally, participation in planned organized sport activities or other training will be noted to standardize physical activity the day before the experimental day. This information will also be used in order to determine which consistent weekday the participant would visit the lab, where they would have performed little or the same level of physical activity before each visit.

After the familiarization visit, allocation sequence for the order of experimental conditions will be assigned through individual randomization from a computergenerated random order, equally assigned to every participant. Randomization will be computer-generated by a researcher involved in the study and will be concealed through a password-protected document that will not be revealed until recruitment and the familiarization visit is completed. Researchers cannot be blinded in this type of study design or condition. Researchers within the study will have access to the allocation sequence list and will retrieve the randomization code prior to each experimental day in order to prepare the laboratory equipment.

\section{Pre-experimental day monitoring}

In order to standardize the $24 \mathrm{~h}$ prior to each test day, participants will be advised to abstain from any heavy physical activity and will be asked to record details about their physical activity and sleep in a standardized diary. Participants will also wear activity monitors during the $24 \mathrm{~h}$ prior to test day to collect data on physical activity and sedentary behavior (hip-worn Actigraph GT3X+ and thigh-worn activPAL micro) and sleep (wrist-worn Actigraph GT3X+). Physical activity and sedentary variables from the Actigraph will include total physical activity and percentages of wear time spent in sedentary, light, and moderate-to-vigorous physical activity, according to standardized cut-points [53]. Physical activity variables from the activPAL micro monitor will include time spent sitting including in prolonged bouts $(>20 \mathrm{~min})$, number of breaks from sitting each hour and time spent standing, stepping, and intense stepping. During night 
time, participants will wear the Actigraph GT3X+ on their left wrist [54]. Using the most common algorithm for sleep-wake scoring in children developed by Sadeh et al. [55], the following key aspects of sleep will be calculated: time to sleep onset, sleep efficiency, times waking up after sleep onset, and total sleep. Diaries will also be filled in to provide complementary details on placement time of activity monitors, sleep timing, and sleep behavior.

In order to standardize dietary intake and blood glucose levels, participants will be asked to record their dietary intake for the $24 \mathrm{~h}$ prior to each condition in a standardized food diary and closely match their dinner for each pre-condition day. They will also be asked to fast and abstain from caffeine after dinner the night before. A researcher will call or message the parents the day before the pre-condition day to remind about physical activity behavior the day before testing, taxi ride, standardizing the dinner, saliva samples, and health state of the participant (e.g., no COVID-19 symptoms). After each laboratory visit, a researcher will call or message to ensure that no influenza symptoms have developed in the household of the participant.

\section{Experimental conditions}

To minimize physical activity on the morning of the visit, participants will be transported to the laboratory via taxi. On the morning of testing, participants will be asked to provide four saliva samples (for cortisol testing); immediately after waking up and at 30,45 , and $60 \mathrm{~min}$ thereafter. Once the participants arrive at the laboratory, a practice session of the cognitive tests will be performed, a saliva sample will be taken, and then a timerestricted, individually standardized breakfast (based on their food choices decided at the familiarization visit) will be provided. Participants at their initial visit will be advised to eat their preferred amount, which will be subsequently weighed and recorded. The portion sizes will be replicated at each visit. The activPAL activity monitor will be worn during the experimental conditions to monitor sitting time during the test day. Participants will be equipped with a chest strap heart rate monitor to be worn during the whole test day visit. After breakfast, pre-condition outcome measures will be collected.

The three experimental conditions (summarized in Fig. 1) are (A) 80-min prolonged sitting with 3-min seated social breaks every 17 min (SOCIAL), (B) 80-min sitting with 3-min bouts of simple resistance activities every 17 min (SRA), or (C) 80-min sitting with 3-min bouts of moderate-intensity stepping every $17 \mathrm{~min}$ (STEP). During sitting, participants will be permitted to read a book, do schoolwork (from a laptop is permitted), and drink water, but not doze off or play games. The SOCIAL break (condition A) will consist of a 3-min chat between a research staff member and the participant while seated. For the SRA break (condition B), participants will be asked to complete 20-s body weight halfsquats, 20-s calf raises, and 20-s gluteal contractions and knee raises repeated three times in sequential order by following standardized video instructions (as per [56]) with music. The STEP break (condition C) will be performed at moderate intensity at a pre-determined pace (around 110 beats per minute), using a standardized step $(27.5 \mathrm{~cm})$ following a standardized video with music in order to achieve a heart rate substantially higher than the SRA break. Toilet breaks and duration of the breaks will be recorded. Heart rate will be measured during the $80 \mathrm{~min}$ intervention at the $2: 50 \mathrm{~min}$ time point during each break, and rate of perceived exertion (RPE) based on the Borg scale [57] will be evaluated immediately following each break. Heart rate will also be assessed once at the start of the first 1-back of the cognitive test (Fig. 2). In order to reduce the spread of COVID-19, certain hygienic and protective regulations and procedures will be followed. Details can be found in Additional file 1 .

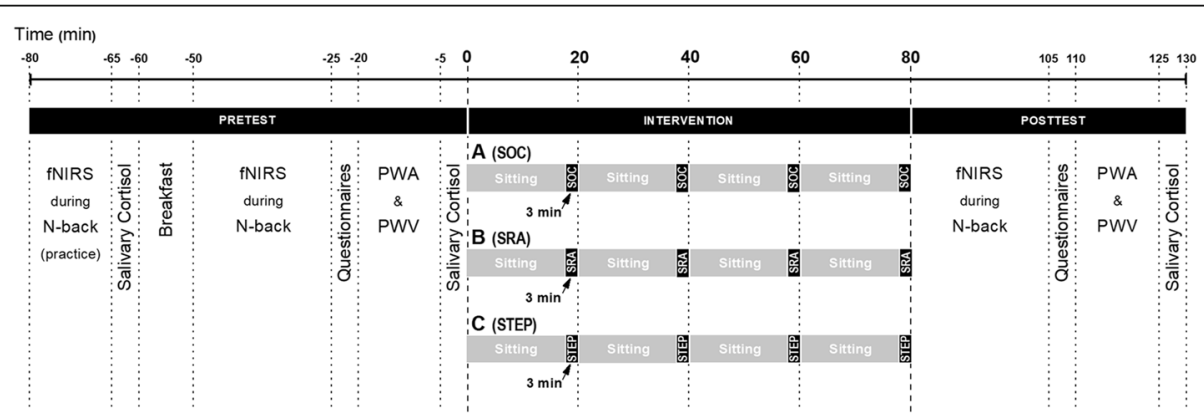

Fig. 1 Experimental day procedures of the three conditions, including pretests, the 80-min intervention, and post-tests. FNIRS will be measured while participants perform working memory tests ( $n$-back). Condition A: prolonged sitting with 3-min seated social breaks every 17 min (SOC); Condition B: prolonged sitting with 3-min simple resistance activities every 17 min (SRA); Condition C: prolonged sitting with 3-min step-up activities every $17 \mathrm{~min}$ (STEP). fNIRS, functional near-infrared spectroscopy; PWA, pulse wave analysis; PWV, pulse wave velocity 

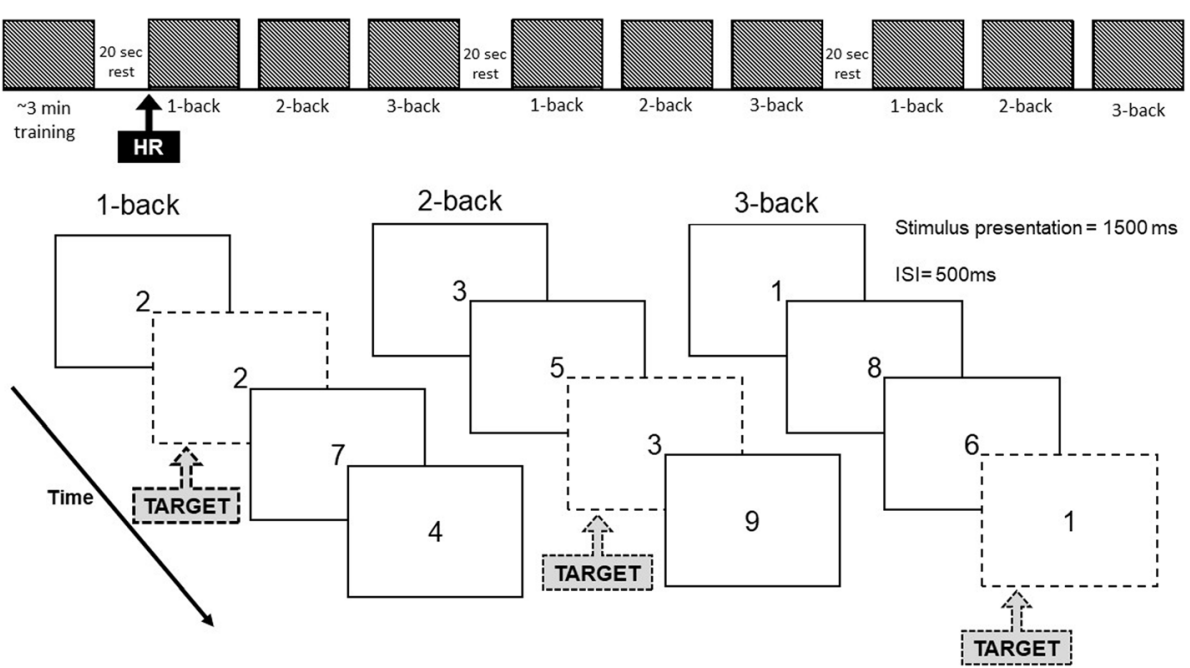

Fig. 2 Schematic of working memory, $n$-back tests that will be administered while wearing the functional near-infrared spectroscopy cap to examine task-related changes in cerebral blood flow. The numerical $n$-back tests will precede with a short training session with feedback. Then 3 blocks of 1, 2, and 3-back tests (each block repeated 3 times), with a 20-s fixation screen between each of the 3 blocks and instructions before each n-back. Examples of correct responses for each n-back are labeled as "Target." Heart rate (HR) will be measured at the start of the first 1 back. ISI, interstimuli interval

\section{Outcome measures}

Outcome measures will be collected immediately prior to and immediately after each experimental condition.

\section{Cerebral blood flow}

The primary outcome measure will be the changes in oxygenated hemoglobin (oxy- $\mathrm{Hb}$ ) using a non-invasive multi-channel continuous wave fNIRS instrument (portable NIRSport, 8-8 system, with short-separation channels, NIRx Medizintechnik GmbH, Berlin, Germany). The optodes will be positioned over the prefrontal cortex, with data sampled at $7.81 \mathrm{~Hz}$ at wavelengths $750 \mathrm{~nm}$ and $820 \mathrm{~nm}$, for oxy-Hb and deoxygenated hemoglobin (deoxy-Hb), respectively. The fNIRS cap has 8 LED light sources and 8 detectors placed according to the standard 10-20 system, with a source-detector separation distance of $3 \mathrm{~cm}$ (Additional file 2). The eighth detector is split into 8 detectors for the additional short-separation optodes with a distance of $0.8 \mathrm{~cm}$ from each source (NIRx Medizintechnik GmbH, Berlin, Germany) to account for superficial blood flow [43, 58]. We chose to measure cerebral blood flow in the prefrontal cortex region as working memory tasks (such as the n-back) predominately activate this region [59-61]. After dimming the lights, and headphones placed on the participant to minimize auditory distractions, system calibration will be performed before each assessment using NIRStar 15.2 software, and using the predefined montage; and the fNIRS signals will be visually quality checked and noted during data collection for motion artifacts or lost channels. Cerebral blood flow measures will be taken simultaneously while the participant performs 3 cognitive tasks of different workloads (numerical 1-, 2-, and 3back tests). Our primary outcome variables will be changes in oxy- $\mathrm{Hb}$ between the 1-back and 2-back, and between the 1-back and 3-back tests. We will also record deoxy-Hb concentrations. The frontal border of the fNIRS cap will be placed about $2 \mathrm{~cm}$ above the nasion point and centered on the $\mathrm{Cz}$, in order to capture prefrontal cortex blood flow changes. We chose to examine oxy-Hb because this signal has been previously shown to correlate with blood flow better than the deoxygenated signal [62], and is more highly correlated with the blood-oxygen-level-dependent (BOLD) fMRI signal [62, 63], although both will be reported.

\section{fNIRS calibration and data processing}

Changes in cerebral blood flow (oxy-Hb) will be assessed using the MATLAB-based software NIRS Brain AnalyzIR toolbox (https://github.com/huppertt/nirs-toolbox) [64]. The data will be predicted on a block design, which is characterized by alternating periods of activity and rest to facilitate the acquisition of the fNIRS signals. Each trial will be preceded by $20 \mathrm{~s}$ of rest. Raw voltage will be converted to optical density, then to hemoglobin concentrations according to the modified Beer-Lambert law. After, the hemodynamic response function will be detected using an autoregressive model-based algorithm to solve for the general linear model (GLM), which allows for residuals of the GLM to be at random [65]. This approach uses pre-whitening filters using autoregressive models and employs iterative reweighted least squares, taking into account serial correlations, motion artifacts, and simultaneous regression of the short-separation 
channels. Gaussian functions will be used with a set standard deviation and separation of means according to the timeframe of the n-back task for the temporal basis of the function. First level statistics will be performed between each source-detector pair between the baseline (1-back) and the task conditions (2- and 3-back). False discovery rate (FDR) correction using a BenjaminiHochberg procedure will be employed to correct for multiple comparisons at a critical level of $<0.05$. Then, second-level group statistics will be performed on the regression coefficients obtained from the first-level statistics. Region-of-interest (ROI) statistics will be carried out to derive averages over all channels.

\section{Cognitive performance}

Cognitive performance in working memory will be assessed using a computerized $n$-back test [66], consisting of the 1-, 2-, and 3-back tests administered simultaneously with fNIRS measures. Participants will be required to indicate (via a key press within $2 \mathrm{~s}$ from stimulus onset) whether the digit presented on the screen was the same digit as the digit presented 1 stimulus previously (1-back), 2 stimuli previously (2-back), or 3 stimuli previously (3-back). Each digit will be presented for $1.5 \mathrm{~s}$ at an interstimuli interval of $500 \mathrm{~ms}$. The n-back tests were created using E-Prime 2.0 (Psychology Software Tools). In order to reduce practice effects, participants will perform a shorter version (2 blocks) of the cognitive tests at the familiarization session and prior to data collection on each experimental day. The outcome variables for cognitive performance will include average reaction time (ms) and accuracy (average number of correct responses) for each test across 3 blocks of 20 digit sequences (see Fig. 2). There will be a 20 -s rest between each set of 3 blocks, involving staring at a dot on the screen while internally counting up from 0 to allow for a short period of cognitive rest after instructions are presented on the screen. Practice tests with feedback will be provided before each testing followed by the actual testing without feedback. A task-based baseline was chosen (1-back), as rest is dynamic across age, and not considered an equalizer in younger age [67].

\section{Arterial stiffness and pulse wave velocity}

Arterial stiffness (defined as augmentation index [AIx]) and pulse wave velocity (PWV) will be measured using a SphygmoCor XCEL PWA/PWV system (AtCor Medical, Sydney, NSW, Australia) [68]. The technology is noninvasive, reproducible, and accurate using the radial pressure waveform to calculate the aortic pressure waveform. The AIx can be derived from the aortic pressure waveform and is defined as the difference between the first and the second systolic peak. PWV is considered the gold standard method for assessing arterial stiffness.
PWV is calculated by the formula: PWV $(\mathrm{m} / \mathrm{s})=$ distance between measurement location $(\mathrm{m}) /$ transit time (s) [69]. First, after 2 min supine rest, blood pressure and AIx will be determined. Then, the distance from the suprasternal notch to the carotid site, from the suprasternal notch to the femoral site (top of the leg cuff), and from the femoral pulse to the top of the leg cuff will be measured using a measuring tape. These distances will be subsequently used to calculate PWV. Three high fidelity pressure waveforms will be recorded using a carotid tonometer with a leg cuff to capture blood pressure waveforms at the carotid and femoral sites. The average of the three high-quality recordings will be used to determine PWV $(\mathrm{m} / \mathrm{s})$.

\section{Other measures}

Stress will be measured using salivary cortisol concentrations. Saliva samples (collected during the first hour after waking up on test days, prior to breakfast, and before and after the 80-min intervention) will be centrifuged for $10 \mathrm{~min}$ at $4{ }^{\circ} \mathrm{C}$ at $2800 \mathrm{rpm}$, and subsequently frozen at $-80{ }^{\circ} \mathrm{C}$. Concentrations will be measured using the ELISA kit Abcam, ab154996. Questionnaires on mood, alertness, and sleepiness that have been previously used in studies with adolescents will be filled-in while seated after fNIRS measurement and prior to the 2-min supine rest before the arterial stiffness measurement. Mood will be assessed using the Positive and Negative Affect Scale (PANAS) [70-72], which includes 10 positive and 10 negative affects. Participants will rate on a 5-point scale (very slightly/not at all; a little; moderately; quite a bit; extremely) the extent to which they experience that mood at the present moment. The positive affect adjectives include interested, excited, strong, enthusiastic, proud, alert, inspired, determined, attentive, and active. The negative mood adjectives include distressed, upset, hostile, irritable, scared, jittery, afraid, ashamed, guilty, and nervous. Alertness will be measured using a simple 10-cm visual analog scale (VAS) [73], going from "not at all" to "completely alert". Sleepiness will be measured using the Karolinska Sleepiness Questionnaire (KSS) [74-76], where participants will rate on a 9-point Likert scale their current level of sleepiness going from "extremely alert" to "very sleepy, great effort to keep awake, fighting sleep".

\section{Data management}

All participants will be assigned a unique identifier in order to remain anonymous. All personal data will be stored in encrypted files with access restricted to study staff. Original paper files will be stored in secure, locked cabinets on site. All data will be entered electronically at GIH. Data entered will be double-checked for errors at the time of entry, and changes made will be documented. 
Additional detection will be performed through the statistical software. Data ranges and consistency checks will be performed for data integrity. No specific auditing procedures are planned for this trial.

\section{Sample size}

Studies with a similar design, and in children and youth are lacking. Thus, the sample size is based on studies of young and older adults. Using the data from these studies [18-20, 31, 77-79] software G*Power (Franz Faul, Universität Kiel, Germany, v 3.1.9.2) was used to calculate effect sizes. Effect sizes between 0.9 and 2.4 for the change in oxygenated hemoglobin were calculated, producing sample sizes between 6 and 13 participants (given an $\alpha=0.05$ and $\beta=0.8$ assuming a two-tailed test). However, in order to have high enough variance within this age group, we will recruit 16 participants.

\section{Statistical methods}

Mixed effects models with subject as a random effect to assess within condition differences will be performed, and to examine time-by-condition interaction effects. Changes in arterial stiffness and PWV will be assessed in relation to cerebral blood flow changes in the mixed models.

Exploratory analyses will be performed excluding the short-separation regressors and ROI averages to compare to the main analyses.

Secondary outcomes (i.e., cognitive function, psychological factors, stress), will be assessed using mixed models, with the subject as a random effect, looking at both the within condition changes and the time-bycondition interaction effects. Correlational analyses will be performed to investigate the association between changes in cognitive function and cerebral blood flow if significant changes are seen. Maximum likelihood estimation will be used in the mixed models to handle missing data at random. Subsequent sensitivity analyses will be performed if necessary.

\section{Discussion}

As interventions to improve school-based performance have become a priority, greater understanding is needed on the effects of physical activity breaks on cognitive performance and the underlying mechanisms. This study will provide insight into the effects of frequent, short physical activity breaks during a prolonged period of sitting on cerebral blood flow. This feasible approach to physical activity breaks during the day may be promising for implementation in schools where children spend most of their time and often in a seated posture. With emerging evidence regarding increasing sedentary behavior and poor academic performance, strategies to break up sitting are needed that are not cumbersome. Furthermore, understanding the underlying mechanisms that may be driving the association can be pivotal so that more informed interventions can be developed and precise recommendations supplied to benefit schoolchildren's health and psychological well-being and reduce sedentary behavior. The results from this study will also tackle some of the lingering questions regarding other intensities, durations, and frequencies of physical activity breaks. Moreover, the effects on psychological well-being will also be investigated, which are important in order to assess adolescents' subjective well-being. Assessing whether differences in arterial stiffness, even at a young age, mediates or moderates cerebral blood flow may add understanding into the mechanisms underpinning effects of prolonged sitting on cerebral blood flow, mood, and working memory performance.

There are many strengths of this trial, such as the use of state-of-the-art technology in measuring cerebral blood flow-fNIRS with short-separation channels. This can give more accurate results that have been lacking in previous studies [31-34, 41, 80]. The mimicking of the school environment (duration of 2 school lessons), and use of devices to measure physical activity and sedentary behavior provide more robust and ecologically valid estimates of pre-day physical activity and sleep behaviors. Additionally, the exercise paradigms are readily available to be implemented in school settings. Although the inclusion of a control group might be considered in a more potent study design, the randomized crossover design is a more attractive alternative as it allows the participants to be their own controls, thus reducing variability between individuals. Mind wandering may affect the participants as well as potential anticipatory affects due to awareness of the condition and subsequent conditions. However, the use of counting during the rest periods and randomization of the order of the conditions will help in minimizing these contaminations. Repositioning of the cap during test days may also reduce accuracy in measurement from pre-test to post-test, however, the use of a marker on the forehead will help to reduce misalignment. In addition, only the prefrontal cortex will be measured in this study, thus cortical activity in other regions cannot be measured. The experiments are to mimic a school day; therefore, generalizability to other settings or days of the week (i.e., weekend) needs to be carefully considered. Finally, this is the first study of this kind therefore subsequent studies will be required to confirm the results.

\section{Trial status}

This is the first version of the protocol. The trial was last amended in the registry December 16, 2020, on https:// clinicaltrials.gov/ct $2 /$ show $/ \mathrm{NCT} 04552626$ ?term $=$ $\mathrm{ABBaH} \&$ draw $=2 \&$ rank $=1$. Recruitment began on September 15, 2020, and was completed in January 
2021. The COVID-19 situation prompted us to urgently begin with recruitment and data collection, thus placing protocol submission alongside said tasks and effectively delayed with regard to our preferred schedule.

\section{Abbreviations}

CBF: Cerebral blood flow; fNIRS: Functional near-infrared spectroscopy; fMRI: Functional magnetic resonance imaging; oxy-Hb: Oxygenated hemoglobin; deoxy-Hb: Deoxygenated hemoglobin; GLM: General linear model; BOLD: Blood-oxygen-level-dependent; ROI: Region-of-interest; Alx: Augmentation index; PWV: Pulse wave velocity; PANAS: Positive and Negative Affect Scale; VAS: Visual analog scale; KSS: Karolinska Sleepiness Scale

\section{Supplementary Information}

The online version contains supplementary material available at https://doi. org/10.1186/s13063-021-05972-5.

$$
\begin{aligned}
& \text { Additional file 1. Laboratory procedures and regulations concerning } \\
& \text { COVID-19 (.pdf) } \\
& \text { Additional file 2. Prefrontal cortex montage setup for the fNIRS cap } \\
& \text { (.pdf) } \\
& \text { Additional file 3. SPIRIT Checklist (.docx) }
\end{aligned}
$$

\section{Acknowledgements}

Not applicable.

\section{Protocol amendments}

Any substantive changes made to the protocol that may influence the study conduct or safety of participants will be formally communicated to the ethics committee, participants, parents, and the journal, accordingly.

\section{Authors' contributions}

ÖE is the project investigator. All authors contributed to designing, developing, and planning the study. EGH drafted the manuscript. All authors contributed to reviewing, revising, and approving the final manuscript and will be involved in the data collection.

\section{Funding}

This project was supported by The Knowledge Foundation (20160040), and the following companies: COOP Sverige, IKEA, Skandia, Skanska, Generation Pep, and Konsumentföreningen Stockholm. Neither the funder nor the partner companies had a role in the design of the study and collection, nor will they in analysis, interpretation of data, or in writing the manuscript. COOP Sverige will provide the breakfasts during the study. Open Access funding provided by Uppsala University.

\section{Availability of data and materials}

Upon reasonable request, data may be made available by contacting the project investigator ÖE.

\section{Declarations}

\section{Ethics approval and consent to participate}

This sub-study obtained ethical approval from the Swedish Ethical Review Authority, Stockholm, Sweden (Dnr 2020-02597). Trial registration was performed retrospectively at www.clinicaltrials.gov (NCT04552626) https:// clinicaltrials.gov/ct2/show/NCT04552626?term $=$ ABBaH\&draw $=2 \&$ rank $=1$ on September 21, 2020, after recruitment began on September 15, 2020. Registration of this study design is becoming more common as it increases good scientific practice [81]. Written informed consent will be received by students and both parents prior to data collection. Provision and compensation will be made in the case of any harm suffered by participants.

This study is part of a larger project called "Physical activity for healthy brain functions in school youth", with the aim to investigate the association between physical activity and healthy brain functions. Thus, the results from this project will be directly used in the planning of interventions that will be implemented in schools in the Stockholm area.

\section{Consent for publication}

Not applicable.

\section{Competing interests}

The authors declare that they have no competing interests.

\section{Author details}

${ }^{1}$ Department of Physical Activity and Health, The Swedish School of Sport and Health Sciences $(\mathrm{GIH})$, Lidingövägen 1, 11433 Stockholm, Sweden. ${ }^{2}$ Department of Surgical Sciences, Medical Epidemiology, Uppsala University, Dag Hammarskjölds väg 14B, 75185 Uppsala, Sweden. ${ }^{3}$ Department of Physiology, Nutrition, and Biomechanics, The Swedish School of Sport and Health Sciences $(\mathrm{GlH})$, Lidingövägen 1, 11433 Stockholm, Sweden. ${ }^{4}$ Department of Global Public Health, Karolinska Institutet, Solnavägen 1, 17177 Solna, Sweden. ${ }^{5}$ Department of Neuroscience, Karolinska Institutet, Solnavägen 1, 17177 Solna, Sweden. 'Division of Insurance Medicine, Department of Clinical Neuroscience, Karolinska Institutet, Solnavägen 1, 17177 Solna, Sweden.

Received: 5 February 2021 Accepted: 23 December 2021

Published online: 06 January 2022

\section{References}

1. Masini A, Marini S, Gori D, Leoni E, Rochira A, Dallolio L. Evaluation of school-based interventions of active breaks in primary schools: a systematic review and meta-analysis. J Sci Med Sport. 2020;23(4):377-84. https://doi. org/10.1016/j.jsams.2019.10.008.

2. Pontifex MB, McGowan AL, Chandler MC, Gwizdala KL, Parks AC, et al. A primer on investigating the after effects of acute bouts of physical activity on cognition. Psychol Sport Exerc. 2019;40:1-22. https://doi.org/10.1016/j. psychsport.2018.08.015.

3. Hillman $\mathrm{CH}, \mathrm{McDonald} \mathrm{KM}$, Logan NE. A review of the effects of physical activity on cognition and brain health across children and adolescence. Nestle Nutr Inst Workshop Ser. 2020:95:1-11. https://doi. org/10.1159/000511508.

4. Nyberg G, Kjellenberg K, Fröberg A, Lindroos AK. A national survey showed low levels of physical activity in a representative sample of Swedish adolescents. Acta Paediatr. 2020;109(11):2342-53.

5. Donnelly JE, Hillman CH, Castelli D, Etnier JL, Lee S, Tomporowski P, et al. Physical activity, fitness, cognitive function, and academic achievement in children: a systematic review. Med Sci Sports Exerc. 2016;48(6):1197-222. https://doi.org/10.1249/MSS.0000000000000901.

6. Ludyga S, Gerber M, Brand S, Holsboer-Trachsler E, Pühse U. Acute effects of moderate aerobic exercise on specific aspects of executive function in different age and fitness groups: a meta-analysis. Psychophysiology. 2016; 53(11):1611-26. https://doi.org/10.1111/psyp.12736.

7. van den Berg V, Saliasi E, Jolles J, de Groot RHM, MJM C, et al. Exercise of varying durations: no acute effects on cognitive performance in adolescents. Front Neuroscience. 2018;12(672).

8. Howie EK, Schatz J, Pate RR. Acute effects of classroom exercise breaks on executive function and math performance: a dose-response study. Res Q Exerc Sport. 2015;86(3):217-24. https://doi.org/10.1080/02701367.2015.103 9892.

9. Singh AS, Saliasi E, van den Berg V, Uijtdewilligen L, de Groot RHM, Jolles J, et al. Effects of physical activity interventions on cognitive and academic performance in children and adolescents: a novel combination of a systematic review and recommendations from an expert panel. $\mathrm{Br} J$ Sports Med. 2019:53(10):640-7. https://doi.org/10.1136/bjsports-2017-098136.

10. de Greeff JW, Bosker RJ, Oosterlaan J, Visscher C, Hartman E. Effects of physical activity on executive functions, attention and academic performance in preadolescent children: a meta-analysis. J Sci Med Sport. 2018;21(5):501-7. https://doi.org/10.1016/j.jsams.2017.09.595.

11. Diamond A. Executive Functions. Annu Rev Psychol. 2013;64(1):135-68. https://doi.org/10.1146/annurev-psych-113011-143750.

12. Lott $M A$, Jensen $C D$. Executive control mediates the association between aerobic fitness and emotion regulation in preadolescent children. J Pediatr Psychol. 2017;42(2):162-73. https://doi.org/10.1093/jpepsy/jsw052.

13. Moriguchi $Y$, Hiraki K. Prefrontal cortex and executive function in young children: a review of NIRS studies. Front Hum Neurosci. 2013;7:867. https:// doi.org/10.3389/fnhum.2013.00867. 
14. Chang YK, Labban JD, Gapin Jl, Etnier JL. The effects of acute exercise on cognitive performance: a meta-analysis. Brain Res. 2012;1453:87-101. https:// doi.org/10.1016/j.brainres.2012.02.068.

15. Baddeley AD, Hitch GJ. Developments in the concept of working memory. Neuropsychology. 1994;8(4):485-93. https://doi.org/10.1037/ 0894-4105.8.4.485.

16. Herold F, Wiegel P, Scholkmann F, Müller NG. Applications of Functional Near-Infrared Spectroscopy (fNIRS) neuroimaging in exercise-cognition science: a systematic. Methodol Focus Rev J Clin Med. 2018;7(12):466. https://doi.org/10.3390/jcm7120466.

17. Hillman CH, Pontifex MB, Raine LB, Castelli DM, Hall EE, Kramer AF. The effect of acute treadmill walking on cognitive control and academic achievement in preadolescent children. Neuroscience. 2009;159(3):1044-54. https://doi.org/10.1016/j.neuroscience.2009.01.057.

18. Byun K, Hyodo K, Suwabe K, Ochi G, Sakairi Y, Kato M, et al. Positive effect of acute mild exercise on executive function via arousal-related prefrontal activations: an fNIRS study. Neuroimage. 2014;98:336-45. https://doi.org/10.1 016/j.neuroimage.2014.04.067.

19. Hyodo K, Dan I, Suwabe K, Kyutoku Y, Yamada Y, Akahori M, et al. Acute moderate exercise enhances compensatory brain activation in older adults. Neurobiol Aging. 2012;33(11):2621-32. https://doi.org/10.1016/j.neurobiola ging.2011.12.022

20. Ichinose Y, Morishita S, Suzuki R, Endo G, Tsubaki A. Comparison of the effects of continuous and intermittent exercise on cerebral oxygenation and cognitive function. Adv Exp Med Biol. 2020;1232:209-14. https://doi.org/10.1 007/978-3-030-34461-0_26.

21. Yanagisawa H, Dan I, Tsuzuki D, Kato M, Okamoto M, Kyutoku Y, et al. Acute moderate exercise elicits increased dorsolateral prefrontal activation and improves cognitive performance with Stroop test. Neuroimage. 2010;50(4): 1702-10. https://doi.org/10.1016/j.neuroimage.2009.12.023.

22. Tari B, Vanhie JJ, Belfry GR, Shoemaker JK, Heath M. Increased cerebral blood flow supports a single-bout postexercise benefit to executive function: evidence from hypercapnia. J Neurophysiol. 2020;124(3):930-40. https://doi.org/10.1152/jn.00240.2020.

23. Moriarty T, Bourbeau K, Bellovary B, Zuhl MN. Exercise intensity influences prefrontal cortex oxygenation during cognitive testing. Behav Sci (Basel). 2019;9(8):83. https://doi.org/10.3390/bs9080083.

24. Murata Y, Watanabe T, Terasawa S, Nakajima K, Kobayashi T, et al. Moderate exercise improves cognitive performance and decreases cortical activation in the go/no-go task. BAOJ Med Nurs. 2015;1(1):002.

25. Salmon J. Novel strategies to promote children's physical activities and reduce sedentary behavior. J Phys Act Health. 2010;7(Suppl 3):S299-306. https://doi.org/10.1123/jpah.7.s3.s299.

26. Carter SE, Draijer R, Holder SM, Brown L, Thijssen DHJ, et al. Effect of different walking break strategies on superficial femoral artery endothelial function. Physiol Rep. 2019;7(16):e14190-e

27. English C, Janssen H, Crowfoot G, Bourne J, Callister R, Dunn A, et al. Frequent, short bouts of light-intensity exercises while standing decreases systolic blood pressure: breaking Up Sitting Time after Stroke (BUST-Stroke) trial. Int I Stroke. 2018;13(9):932-40. https://doi.org/10.1177/1747493018798535.

28. Loh R, Stamatakis E, Folkerts D, Allgrove JE, Moir HJ. Effects of interrupting prolonged sitting with physical activity breaks on blood glucose, insulin and triacylglycerol measures: a systematic review and meta-analysis. Sports Med. 2020;50(2):295-330. https://doi.org/10.1007/s40279-019-01183-w.

29. Yates T, Edwardson CL, Celis-Morales C, Biddle SJH, Bodicoat D, Davies MJ, et al. Metabolic effects of breaking prolonged sitting with standing or light walking in older South Asians and White Europeans: a randomized acute study. J Gerontol A Biol Sci Med Sci. 2020;75(1):139-46. https://doi.org/10.1 093/gerona/gly252.

30. Altenburg TM, Rotteveel J, Dunstan DW, Salmon J, Chinapaw MJ. The effect of interrupting prolonged sitting time with short, hourly, moderate-intensity cycling bouts on cardiometabolic risk factors in healthy, young adults. J Appl Physiol. 1985;115(12):1751-6.

31. Carter SE, Draijer R, Holder SM, Brown L, Thijssen DHJ, et al. Regular walking breaks prevent the decline in cerebral blood flow associated with prolonged sitting. J Appl Physiol. 1985;125(3):790-8.

32. Maasakkers CM, Melis RJF, Kessels RPC, Gardiner PA, Olde Rikkert MGM, Thijssen $\mathrm{DHJ}$, et al. The short-term effects of sedentary behaviour on cerebral hemodynamics and cognitive performance in older adults: a cross-over design on the potential impact of mental and/or physical activity. Alzheimers Res Ther. 2020;12(1):76. https://doi.org/10.1186/s13195-020-00644-z.
33. Perdomo SJ, Gibbs BB, Kowalsky RJ, Taormina JM, Balzer JR. Effects of alternating standing and sitting compared to prolonged sitting on cerebrovascular hemodynamics. Sport Sci Health. 2019;15(2):375-83.

34. Wheeler MJ, Dunstan DW, Smith B, Smith KJ, Scheer A, et al. Morning exercise mitigates the impact of prolonged sitting on cerebral blood flow in older adults. J Appl Physiol. 1985;126(4):1049-55.

35. Chrismas BCR, Taylor L, Cherif A, Sayegh S, Bailey DP. Breaking up prolonged sitting with moderate-intensity walking improves attention and executive function in Qatari females. PLoS ONE. 2019;14(7):e0219565. https://doi.org/10.1371/journal.pone.0219565.

36. Mullane SL, Buman MP, Zeigler ZS, Crespo NC, Gaesser GA. Acute effects on cognitive performance following bouts of standing and light-intensity physical activity in a simulated workplace environment. J Sci Med Sport. 2017;20(5):489-93. https://doi.org/10.1016/j.jsams.2016.09.015.

37. Wennberg P, Boraxbekk CJ, Wheeler M, Howard B, Dempsey PC, Lambert G, et al. Acute effects of breaking up prolonged sitting on fatigue and cognition: a pilot study. BMJ Open. 2016;6(2):e009630. https://doi.org/10.113 6/bmjopen-2015-009630

38. Wheeler MJ, Green DJ, Ellis KA, Cerin E, Heinonen I, Naylor LH, et al. Distinct effects of acute exercise and breaks in sitting on working memory and executive function in older adults: a three-arm, randomised cross-over trial to evaluate the effects of exercise with and without breaks in sitting on cognition. Br J Sports Med. 2020;54(13):776-81. https://doi.org/10.1136/ bjsports-2018-100168.

39. Neubauer AC, Fink A. Intelligence and neural efficiency. Neurosci Biobehav Rev. 2009:33(7):1004-23. https://doi.org/10.1016/j.neubiorev.2009.04.001.

40. Heiland EG, Tarassova O, Fernström M, English C, Ekblom Ö, Ekblom MM. Frequent, short physical activity breaks reduce prefrontal cortex activation but preserve working memory in middle-aged adults: ABBaH study. Front Hum Neurosci. 2021;15:719509. https://doi.org/10.3389/fnhum.2021.719509.

41. Bremer E, Graham JD, Heisz JJ, Cairney J. Effect of acute exercise on prefrontal oxygenation and inhibitory control among male children with autism spectrum disorder: an exploratory study. Front Behav Neurosci. 2020; 14:84. https://doi.org/10.3389/fnbeh.2020.00084.

42. Lambrick D, Stoner L, Grigg R, Faulkner J. Effects of continuous and intermittent exercise on executive function in children aged 8-10 years. Psychophysiology. 2016;53(9):1335-42. https://doi.org/10.1111/psyp.12688.

43. Santosa H, Zhai X, Fishburn F, Sparto P, Huppert T. Quantitative comparison of correction techniques for removing systemic physiological signal in functional near-infrared spectroscopy studies. Neurophotonics. 2020;7(3): 035009. https://doi.org/10.1117/1.NPh.7.3.035009.

44. Altenburg TM, Chinapaw MJ, Singh AS. Effects of one versus two bouts of moderate intensity physical activity on selective attention during a school morning in Dutch primary schoolchildren: a randomized controlled trial. J Sci Med Sport. 2016;19(10):820-4. https://doi.org/10.1016/j.jsams.2015.12.003.

45. Booth JN, Chesham RA, Brooks NE, Gorely T, Moran CN. A citizen science study of short physical activity breaks at school: improvements in cognition and wellbeing with self-paced activity. BMC Med. 2020;18(1):62. https://doi. org/10.1186/s12916-020-01539-4.

46. Sperlich B, De Clerck I, Zinner C, Holmberg HC, Wallmann-Sperlich B. Prolonged sitting interrupted by 6-min of high-intensity exercise: circulatory, metabolic, hormonal, thermal, cognitive, and perceptual responses. Front Physiol. 2018:9:1279. https://doi.org/10.3389/fphys.2018.01279.

47. Veijalainen A, Tompuri T, Haapala EA, Viitasalo A, Lintu N, Väistö J, et al. Associations of cardiorespiratory fitness, physical activity, and adiposity with arterial stiffness in children. Scand J Med Sci Sports. 2016;26(8):943-50. https://doi.org/10.1111/sms.12523.

48. Credeur DP, Miller SM, Jones R, Stoner L, Dolbow DR, Fryer SM, et al. Impact of prolonged sitting on peripheral and central vascular health. Am J Cardiol. 2019;123(2):260-6. https://doi.org/10.1016/j.amjcard.2018.10.014.

49. Ketelhut S, Ketelhut SR, Ketelhut K. School-based exercise intervention improves blood pressure and parameters of arterial stiffness in children: a randomized controlled trial. Pediatr Exerc Sci. 2020;33(1):1-7. https://doi. org/10.1123/pes.2020-0053.

50. Chuensiri N, Tanaka H, Suksom D. The acute effects of supramaximal highintensity intermittent exercise on vascular function in lean vs. obese prepubescent boys. Pediatr Exerc Sci. 2015;27(4):503-9. https://doi.org/1 0.1123/pes.2015-0100.

51. Scharfen J, Jansen $\mathrm{K}$, Holling $H$. Retest effects in working memory capacity tests: A meta-analysis. Psychon Bull Rev. 2018;25(6):2175-99. https://doi. org/10.3758/s13423-018-1461-6. 
52. Collie A, Maruff P, Darby DG, McStephen M. The effects of practice on the cognitive test performance of neurologically normal individuals assessed at brief test-retest intervals. J Int Neuropsychol Soc. 2003;9(3):419-28. https:// doi.org/10.1017/S1355617703930074.

53. Evenson KR, Catellier DJ, Gill K, Ondrak KS, McMurray RG. Calibration of two objective measures of physical activity for children. J Sports Sci. 2008;26(14): 1557-65. https://doi.org/10.1080/02640410802334196.

54. Cellini N, Buman MP, McDevitt EA, Ricker AA, Mednick SC. Direct comparison of two actigraphy devices with polysomnographically recorded naps in healthy young adults. Chronobiol Int. 2013;30(5):691-8. https://doi. org/10.3109/07420528.2013.782312.

55. Sadeh A, Sharkey KM, Carskadon MA. Activity-based sleep-wake identification: an empirical test of methodological issues. Sleep. 1994;17(3): 201-7. https://doi.org/10.1093/sleep/17.3.201.

56. Dempsey PC, Blankenship JM, Larsen RN, Sacre JW, Sethi P, Straznicky NE, et al. Interrupting prolonged sitting in type 2 diabetes: nocturnal persistence of improved glycaemic control. Diabetologia. 2017;60(3):499507. https://doi.org/10.1007/s00125-016-4169-z.

57. Borg G. Borg's perceived exertion and pain scales. Champaign, IL, vol. viii. US: Human Kinetics; 1998. p. 104-viii.

58. Yucel MA, Selb J, Aasted CM, Petkov MP, Becerra L, et al. Short separation regression improves statistical significance and better localizes the hemodynamic response obtained by near-infrared spectroscopy for tasks with differing autonomic responses. Neurophotonics. 2015;2(3):035005. https://doi.org/10.1117/1.NPh.2.3.035005.

59. Owen AM, McMillan KM, Laird AR, Bullmore E. N-back working memory paradigm: a meta-analysis of normative functional neuroimaging studies. Hum Brain Mapp. 2005;25(1):46-59. https://doi.org/10.1002/hbm.20131.

60. Smith EE, Jonides J. Working memory: a view from neuroimaging. Cogn Psychol. 1997;33(1):5-42. https://doi.org/10.1006/cogp.1997.0658.

61. Hoshi Y, Tsou BH, Billock VA, Tanosaki M, Iguchi Y, Shimada M, et al. Spatiotemporal characteristics of hemodynamic changes in the human lateral prefrontal cortex during working memory tasks. Neuroimage. 2003; 20(3):1493-504. https://doi.org/10.1016/\$1053-8119(03)00412-9.

62. Cui X, Bray S, Bryant DM, Glover GH, Reiss AL. A quantitative comparison of NIRS and fMRI across multiple cognitive tasks. Neuroimage. 2011;54(4):280821. https://doi.org/10.1016/j.neuroimage.2010.10.069.

63. Strangman G, Culver JP, Thompson JH, Boas DA. A quantitative comparison of simultaneous BOLD $\mathrm{fMRI}$ and NIRS recordings during functional brain activation. Neuroimage. 2002;17(2):719-31. https://doi.org/10.1006/nimg.2 002.1227.

64. Santosa H, Zhai X, Fishburn F, Huppert T. The NIRS Brain AnalyzIR Toolbox. Algorithms. 2018;11(5):73. https://doi.org/10.3390/a11050073.

65. Barker JW, Aarabi A, Huppert TJ. Autoregressive model based algorithm for correcting motion and serially correlated errors in fNIRS. Biomed Opt Express. 2013;4(8):1366-79. https://doi.org/10.1364/BOE.4.001366.

66. Kirchner WK. Age differences in short-term retention of rapidly changing information. J Exp Psychol. 1958;55(4):352-8. https://doi.org/1 $0.1037 / h 0043688$.

67. Camacho MC, Quiñones-Camacho LE, Perlman SB. Does the child brain rest?: An examination and interpretation of resting cognition in developmental cognitive neuroscience. Neuroimage. 2020;212:116688 https://doi.org/10.1016/j.neuroimage.2020.116688.

68. Hwang MH, Yoo JK, Kim HK, Hwang CL, Mackay K, Hemstreet O, et al. Validity and reliability of aortic pulse wave velocity and augmentation index determined by the new cuff-based SphygmoCor Xcel. J Human Hypertens. 2014;28(8):475-81. https://doi.org/10.1038/jhh.2013.144.

69. Mackenzie IS, Wilkinson IB, Cockcroft JR. Assessment of arterial stiffness in clinical practice. Qjm. 2002;95(2):67-74. https://doi.org/10.1093/qjmed/95.2.67.

70. Crawford JR, Henry JD. The positive and negative affect schedule (PANAS): construct validity, measurement properties and normative data in a large non-clinical sample. Br J Clin Psychol. 2004;43(Pt 3):245-65. https://doi.org/1 0.1348/0144665031752934.

71. Huebner ES, Dew T. Preliminary validation of the positive and negative affect schedule with adolescents. J Psychoeduc Assess. 1995;13(3):286-93. https://doi.org/10.1177/073428299501300307.

72. Lonigan CJ, Hooe ES, David CF, Kistner JA. Positive and negative affectivity in children: confirmatory factor analysis of a two-factor model and its relation to symptoms of anxiety and depression. J Consult Clin Psychol. 1999;67(3):374-86. https://doi.org/10.1037/0022-006X.67.3.374.
73. Monk TH. A Visual Analogue Scale technique to measure global vigor and affect. Psychiatry Res. 1989;27(1):89-99. https://doi.org/10.1016/0165-1781 (89)90013-9.

74. Putilov AA, Donskaya OG. Construction and validation of the EEG analogues of the Karolinska sleepiness scale based on the Karolinska drowsiness test. Clin Neurophysiol Off J Int Fed Clin Neurophysiol. 2013;124(7):1346-52. https://doi.org/10.1016/j.clinph.2013.01.018.

75. Campbell IG, Burright CS, Kraus AM, Grimm KJ, Feinberg I. Daytime sleepiness increases with age in early adolescence: a sleep restriction doseresponse study. Sleep. 2017;40(5):zsx046.

76. Louca M, Short MA. The effect of one night's sleep deprivation on adolescent neurobehavioral performance. Sleep. 2014;37(11):1799-807. https://doi.org/10.5665/sleep.4174.

77. Bediz CS, Oniz A, Guducu C, Ural Demirci E, Ogut H, Gunay E, et al. Acute supramaximal exercise increases the brain oxygenation in relation to cognitive workload. Front Hum Neurosci. 2016;10(174):174. https://doi.org/1 0.3389/fnhum.2016.00174.

78. Endo K, Matsukawa K, Liang N, Nakatsuka C, Tsuchimochi H, Okamura H, et al. Dynamic exercise improves cognitive function in association with increased prefrontal oxygenation. The journal of physiological sciences : JPS. 2013;63(4):287-98. https://doi.org/10.1007/s12576-013-0267-6.

79. Kujach S, Byun K, Hyodo K, Suwabe K, Fukuie T, Laskowski R, et al. A transferable high-intensity intermittent exercise improves executive performance in association with dorsolateral prefrontal activation in young adults. Neuroimage. 2018;169:117-25. https://doi.org/10.1016/j.neuroimage.2 017.12.003.

80. Mehta RK, Shortz AE, Benden ME. Standing up for learning: a pilot investigation on the neurocognitive benefits of stand-biased school desks. Int J Environ Res Public Health. 2015;13(1):ijerph13010059.

81. English C, Weerasekara I, Carlos A, Chastin S, Crowfoot G, Fitzsimons C, et al. Investigating the rigour of research findings in experimental studies assessing the effects of breaking up prolonged sitting - extended scoping review. Braz J Phys Ther. 2020;25(1):4-16. https://doi.org/10.1016/j.bjpt.2020. 04.007.

\section{Publisher's Note}

Springer Nature remains neutral with regard to jurisdictional claims in published maps and institutional affiliations.
Ready to submit your research? Choose BMC and benefit from:

- fast, convenient online submission

- thorough peer review by experienced researchers in your field

- rapid publication on acceptance

- support for research data, including large and complex data types

- gold Open Access which fosters wider collaboration and increased citations

- maximum visibility for your research: over $100 \mathrm{M}$ website views per year

At $\mathrm{BMC}$, research is always in progress.

Learn more biomedcentral.com/submissions 